\title{
Special Killing forms on toric Sasaki-Einstein manifolds
}

\author{
Vladimir Slesar*1, Mihai Visinescu ${ }^{\dagger 2}$, and Gabriel Eduard Vîlcu ${ }^{\ddagger \S 3,4}$ \\ ${ }^{1}$ Department of Mathematics, University of Craiova, \\ Str. Al.I. Cuza, Nr. 13, Craiova 200585, Romania \\ ${ }^{2}$ Department of Theoretical Physics, \\ National Institute for Physics and Nuclear Engineering, \\ Magurele, P.O.Box M.G.-6, Romania \\ ${ }^{3}$ Department of Mathematical Modelling, \\ Economic Analysis and Statistics, \\ Petroleum-Gas University of Ploieşti, \\ Bulevardul Bucureşti, Nr. 39, Ploieşti, 100680, Romania \\ ${ }^{4}$ Faculty of Mathematics and Computer Science, \\ Research Center in Geometry, Topology and Algebra, \\ University of Bucharest, Str. Academiei, Nr. 14, Sector 1, \\ Bucharest, 060042, Romania
}

October 29, 2018

\begin{abstract}
In this paper we study the interplay between complex coordinates on the Calabi-Yau metric cone and the special Killing forms on the toric Sasaki-Einstein manifold. In the general case we give a procedure to locally construct the special Killing forms. In the final part we exemplify the general scheme in the case of the 5-dimensional $Y^{p, q}$ spaces, identifying the additional special Killing 2 -forms which were previously obtained by the second author of the present paper, but with a different method, in [Mod. Phys. Lett. A 27 (2012) 1250217].
\end{abstract}

Keywords:Killing forms; toric manifolds; Sasaki-Einstein manifolds. Mathematics Subject Classification (2010): 53C15; 53C25; 81T20.

PACS Nos.: 04.50.Gh, 04.62.+v .

*vlslesar@central.ucv.ro

${ }^{\dagger}$ mvisin@theory.nipne.ro

‡gvilcu@upg-ploiesti.ro

§vilcu@gta.math.unibuc.ro 


\section{Introduction}

Historically, Sasakian structures grew out of research in contact geometry and were studied extensively in the 1960's. In the last time, there has been renewed interest in Sasaki geometries in connection with some recent developments in mathematics and theoretical physics [21]. New explicit inhomogeneous SasakiEinstein metrics in all dimensions have been constructed in [9]. Moreover, using the construction of Boyer and Galicki [3], infinitely many toric Sasaki-Einstein manifolds with arbitrarily high second Betti number of every dimension $\geq 5$ were given in [25].

A particularly interesting class of toric contact structures on $S^{2} \times S^{3}$ have been studied by physicists [8, 15] and denoted by $Y^{p, q}$ where $p, q$ are relative prime integers satisfying $0<q<p$. These structures have become of much interest in connection with the AdS/CFT conjecture [14]. The AdS/CFT correspondence provides a detailed correspondence between certain conformal field theories and geometries leading to remarkable new results in both sides. The Sasaki-Einstein manifolds with their explicit metrics offer useful models to test AdS/CFT duality.

The purpose of this paper is the explicit construction of the special Killing forms on toric Sasaki-Einstein manifolds, this goal being achieved using foliated coordinates on the metric cone of the manifold. The interest in the Killing forms is motivated by the key role of symmetries in physics. For the geodesic motions in a space-time, the usual conserved quantities are related to the isometries which correspond to Killing vectors. Sometimes a space-time could admit higher order symmetries described by symmetric Stäckel-Killing tensors. These symmetries are known as hidden symmetries, and the corresponding conserved quantities are quadratic or, more general, polynomial in momenta.

Another natural generalization of the Killing vector fields is represented by the Killing forms which in many aspects are more important than the StäckelKilling tensors. It was realized the significant connection between Killing forms and nonstandard supersymmetries. Investigation of the properties of higher dimensional space-times has pointed out the role of conformal Killing forms to generate black holes solutions [6].

In the case of Sasaki-Einstein spaces, there are special Killing forms directly constructed from the contact form of the Sasaki manifolds. Besides these special Killing forms, there are two special Killing forms connected with the additional parallel forms of the Calabi-Yau cone manifolds given by the complex volume and its conjugate [20].

Concerning special Killing forms, we would like to point out that this notion was originally introduced by Tachibana and $\mathrm{Yu}$ in [23], but the concept was mainly developed by Semmelman [20, who gave some equivalent definitions more natural than the original one, and a complete description of manifolds admitting special Killing forms. It is easy to remark that the restriction from Killing forms to special Killing forms is analogous to the definition of a Sasakian structure as a unit Killing vector field satisfying an additional equation. We also note that special Killing forms are of great interest in physics, due to potential 
applications to gauge/gravity duality and string theory. For example, very recently, Houri, Takeuchi and Yasui [12] introduced the concept of a Sasaki manifold with torsion and proved that these metrics admit two types of hidden symmetries, the first arising from the special Killing forms. Moreover, they showed that these metrics on the five-dimensional compact manifolds naturally generalize the toric Sasaki-Einstein metrics $Y^{p, q}$ and $L^{a b c}$.

The great importance of special Killing forms in our setting comes also from the fact that any Killing form of degree at least 3 on a compact Sasaki-Einstein manifold is actually special [22]. In particular, since 3-Sasakian manifolds are automatically Einstein [13, the above result extends on compact 3-Sasakian spaces, an interesting class of manifolds investigated in the context of the AdS/CFT-correspondence by Yee [28, Martelli, Sparks and Yau [18].

The organization of the paper is as follows: In the next Section we give some mathematical preliminaries regarding the special Killing forms on SasakiEinstein manifolds and their Calabi-Yau cones. In order to construct the special Killing forms on Sasaki-Einstein manifolds, in Section 3 we investigate the complex volume and the foliated coordinates on the metric cone, and then we give a procedure to locally extract the special Killing forms on a toric Sasaki-Einstein manifold. In Section 4 we exemplify the general scheme in the case of the $Y^{p, q}$ manifolds, identifying two additional special Killing 2 -forms. We note that these Killing forms were also obtained in [22] with a different approach, namely by a direct calculation. Finally we give our conclusions in Section 5 .

\section{Preliminaries}

The conformal Killing-Yano tensors provide a generalization of Killing vector fields. In order to introduce this concept, we start out by considering the tensor product space $V^{*} \otimes \bigwedge^{p} V^{*}$, where $(V,\langle\cdot, \cdot\rangle)$ is an $m$ - dimensional Euclidean space. Considering the wedge product $\wedge$ and the contraction map $\lrcorner$, this tensor product splits as a direct sum of three terms, with the following identification (see [20])

$$
V^{*} \otimes \bigwedge^{p} V^{*} \simeq \bigwedge^{p+1} V^{*} \oplus \bigwedge^{p-1} V^{*} \oplus \bigwedge^{p, 1} V^{*}
$$

Let us denote by $\operatorname{pr}^{p} \wedge^{p, 1}, \operatorname{pr} \wedge^{p+1}, \operatorname{pr}_{\wedge^{p-1}}$ the projections on the first, second and third term, respectively.

The following obvious relation is satisfied

$$
\operatorname{pr}_{\bigwedge^{p, 1}}=\mathrm{Id}-\operatorname{pr}_{\bigwedge^{p+1}}-\operatorname{pr}_{\bigwedge^{p-1}}
$$

so if $\left\{e_{i}\right\}_{1 \leq i \leq m}$ is an orthonormal basis and $\left\{\alpha^{i}\right\}_{1 \leq i \leq m}$ the associated dual basis, then the above projection can be written as

$$
\begin{aligned}
\operatorname{pr}_{\wedge^{p, 1}}(\alpha \otimes \Psi)= & \left.\alpha \otimes \Psi-\frac{1}{p+1} \sum_{i} \alpha^{i} \otimes e_{i}\right\lrcorner(\alpha \wedge \Psi) \\
& \left.-\frac{1}{n-p+1} \sum_{i} \alpha^{i} \otimes \alpha^{i} \wedge\left(\alpha^{\sharp}\right\lrcorner \Psi\right) .
\end{aligned}
$$


for any $\alpha \in V^{*}$ and $\Psi \in \wedge^{p} V^{*}, \sharp$ being the "musical" isomorphism between $V^{*}$ and $V$, related to the Euclidean structure. We recall that given an element $\alpha \in V^{*}$, we know that there exists a unique $v \in V$ such that $\alpha(u)=\langle v, u\rangle$ for all $u \in V$ and the "sharp" operator $\sharp$ is defined by $\alpha^{\sharp}=v$.

It turns out that $\bigwedge^{p, 1} V^{*}$ is the intersection of the kernels of wedge product and contraction map. This fact can be also checked directly, by considering

$$
\begin{gathered}
\mu_{1}: V^{*} \otimes \bigwedge_{p}^{p} V^{*} \rightarrow \bigwedge_{p-1}^{p+1} V^{*}, \mu_{1}(\alpha \otimes \Psi):=\alpha \wedge \Psi, \\
\left.\mu_{2}: V^{*} \otimes \bigwedge^{*} \rightarrow \bigwedge^{*} V^{*}, \mu_{2}(\alpha \otimes \Psi):=\alpha^{\sharp}\right\lrcorner \Psi,
\end{gathered}
$$

and calculating

$$
\mu_{1} \circ \operatorname{pr}_{\bigwedge^{p, 1}}=0, \mu_{2} \circ \operatorname{pr}_{\bigwedge^{p, 1}}=0 .
$$

Now, let us consider a Riemannian manifold $(M, g)$ with $\operatorname{dim} M=m$ endowed with the Levi-Civita connection $\nabla$. If $\Psi \in \Gamma\left(\bigwedge^{p} T M^{*}\right)$ is a smooth differential form of dimension $p$, then we can regard $\nabla \Psi$ as a section in a tensorial fibre bundle, $\nabla \Psi \in \Gamma\left(T M^{*}\right) \otimes \Gamma\left(\bigwedge^{p} T M^{*}\right)$, and we have

$$
\nabla \Psi=\sum_{i} \alpha^{i} \otimes \nabla_{e_{i}} \Psi
$$

where $\left\{e_{i}\right\}_{1 \leq i \leq m}$ and $\left\{\alpha^{i}\right\}_{1 \leq i \leq m}$ are now the corresponding local orthonormal frames.

The twistor operator $T$ is defined as the composition [20]

$$
T:=\operatorname{pr}_{\wedge^{p, 1}} \circ \nabla
$$

and using (1) and (2), we obtain

$$
\begin{aligned}
T(\Psi):= & \operatorname{pr}_{\wedge^{p, 1}}(\nabla \Psi) \\
= & \left.\sum_{i} \alpha^{i} \otimes \nabla_{e_{i}} \Psi-\frac{1}{p+1} \sum_{i} \alpha^{i} \otimes e_{i}\right\lrcorner\left(\sum_{j} \alpha^{j} \wedge \nabla_{e_{j}} \Psi\right) \\
& \left.+\frac{1}{n-p+1} \sum_{i} \alpha^{i} \otimes \alpha^{i} \wedge\left(-\sum_{j} e_{j}\right\lrcorner \nabla_{e j} \Psi\right) \\
= & \left.\sum_{i} \alpha^{i} \otimes\left(\nabla_{e_{i}} \Psi-\frac{1}{p+1} e_{i}\right\lrcorner d \Psi+\frac{1}{n-p+1} \alpha^{i} \wedge d^{*} \Psi\right)
\end{aligned}
$$

where $d$ and $d^{*}$ are the de Rham differential and co-differential operators with respect to the Riemannian structure $g$.

It is now obvious that $T(\Psi)=0$ if and only if

$$
\left.\nabla_{X} \Psi-\frac{1}{p+1} X\right\lrcorner d \Psi+\frac{1}{n-p+1} X^{*} \wedge d^{*} \Psi=0
$$


for any vector field $X$. The differential forms that satisfy the above equation are called conformal Killing -Yano tensors or conformal Killing forms [20].

Coclosed conformal Killing form are called Killing forms. If the dimension is 1 , then Killing forms are just dual to Killing vector fields.

Any parallel form is a Killing form. It is easy also to see that on $\left(\mathbb{R}^{3}, x^{1}, x^{2}, x^{3}\right)$ with canonical metric, the differential form

$$
\Psi=x^{3} d x^{1} \wedge d x^{2}+x^{1} d x^{2} \wedge d x^{3}+x^{2} d x^{3} \wedge d x^{1}
$$

is a Killing form. Other non-trivial Killing and conformal Killing form can be defined on standard sphere, on Sasakian manifolds, on nearly Kähler manifolds.

A particular class of Killing forms is represented by the special Killing forms, which satisfy, for some constant $c$, the equation [23, 20]

$$
\nabla_{X}(d \Psi)=c X^{*} \wedge \Psi
$$

Here $X$ is an arbitrary vector field on $M$. The interesting feature of special Killing forms comes from the fact that the most known Killing forms are actually special.

In order to classify this type of differential forms, in [20. Semmelmann introduced a correspondence between special Killing forms defined on the manifold $M$ and parallel forms defined on the metric cone $C(M)$. This is in fact the product manifold $M \times \mathbb{R}_{>0}$, with $\operatorname{dim} C(M)=2 n=m+1$, endowed with the warped metric $g_{\text {cone }}:=d r^{2}+r^{2} g$. More exactly, a $p$-dimensional differential form $\Psi$ is a special Killing form on $M$ if and only if the corresponding form

$$
\Psi_{\text {cone }}:=r^{p} d r \wedge \Psi+\frac{r^{p+1}}{p+1} d \Psi
$$

is parallel on $C(M)$.

A particular example when the special Killing forms are known to exist is represented by Sasaki-Einstein manifolds. It is well-known that a Sasakian manifold is a Riemannian manifold $(M, g)$ whose metric cone $C(M)$ is Kähler 2]. From this it follows that $M$ also has a contact structure $(\phi, \xi, \eta)$ such that the 1-dimensional foliation generated by the Reeb vector field $\xi$ is transversely Kähler [7]. A Sasaki-Einstein manifold is a Riemannian manifold $(M, g)$ that is both Sasakian and Einstein. On the other hand, a toric Sasakian manifold is a Sasakian manifold $(M, g)$ of dimension $2 n-1$ with Sasakian structure $(\phi, \xi, \eta)$, such that there is an effective action of $n$-dimensional torus $G$, preserving the Sasakian structure, while $\xi$ is an element of the Lie algebra of $G$ [5]. Equivalently, a toric Sasakian manifold is a Sasakian manifold whose Kähler cone is a toric Kähler manifold [10, 11].

On a $(2 n-1)$-dimensional Sasaki manifold with the Reeb vector field $\xi$ and $1-$ form $\eta:=\xi^{*}$, there are the following special Killing forms:

$$
\Psi_{k}=\eta \wedge(d \eta)^{k} \quad, \quad k=0,1, \cdots, n-1 .
$$


Besides these Killing forms, there are $n-1$ closed conformal Killing forms (also called $*$-Killing forms)

$$
\Phi_{k}=(d \eta)^{k} \quad, \quad k=1, \cdots, n-1 .
$$

In the case of a Sasaki-Einstein manifold the metric cone is Ricci-flat, has holonomy $S U_{n}$ and there are two additional parallel forms related to complex volume form. To wit, on this type of manifolds with $S U_{n}$ geometric structure, there exists a complex volume form $\Omega$ and also its conjugate $\bar{\Omega}$, these globally defined complex $n$-dimensional differential forms being furthermore parallel [19, 20, 27]. Then, special real parallel differential forms on the Sasaki-Einstein metric can be derived.

From the above consideration, it becomes clear the relevance of the complex volume form of the metric cone of a Sasaki-Einstein manifold. Consequently, it is of interest to consider in the following the complex coordinates defined on a toric Sasaki-Einstein manifold. The geometric cone of this manifold can be regarded in the classical manner [1, 15, 17.

First of all, we introduce symplectic (action-angle) coordinates $\left(y^{i}, \phi^{i}\right)$; the angular coordinates $\phi^{i}$ will generate the toric action. The corresponding Kähler metric on $C(M)$ will be 17

$$
d s^{2}=G_{i j} d y^{i} d y^{j}+G^{i j} d \phi^{i} d \phi^{j},
$$

where the metric coefficients are obtained using the symplectic potential $G$

$$
G_{i j}=\frac{\partial^{2} G}{\partial y^{i} \partial y^{j}},
$$

and $\left(G^{i j}\right)=\left(G_{i j}\right)^{-1}$.

We can now express the complex structure

$$
J=\left(\begin{array}{cc}
0 & -G^{i j} \\
G_{i j} & 0
\end{array}\right),
$$

while the Kähler form is

$$
\omega=d y^{i} \wedge d \phi^{i} .
$$

Next, we present the complex approach. From symplectic coordinate we can pass to the coordinate patch $\left(x^{i}, \phi^{i}\right)$, obtained from complex coordinates $z^{i}:=x^{i}+\mathrm{i} \phi^{i}$, with $\mathrm{i}:=\sqrt{-1}$. This time, the metric structure is written in the following manner

$$
d s^{2}=F_{i j} d x^{i} d x^{j}+F_{i j} d \phi^{i} d \phi^{j} .
$$

In this setting, the metric coefficients are again obtain using the Hessian of the Kähler potential $F$, i.e.

$$
F_{i j}=\frac{\partial^{2} F}{\partial x^{i} \partial x^{j}} .
$$


With respect to the coordinates $\left(x^{i}, \phi^{i}\right)$, the Kähler form is

$$
\omega=\left(\begin{array}{cc}
0 & F_{i j} \\
-F_{i j} & 0
\end{array}\right) .
$$

The symplectic potential $G$ and the Kähler potential $F$ are related by the Legendre transform

$$
F(x)=\left(y^{i} \frac{\partial G}{\partial y^{i}}-g\right)(y=\partial F / \partial x) .
$$

Consequently, $F$ and $G$ are Legendre dual to each other

$$
F(x)+G(y)=\sum_{j} \frac{\partial F}{\partial x^{j}} \frac{\partial G}{\partial y^{i}} \text { at } x^{i}=\frac{\partial G}{\partial y^{i}} \text { or } y^{i}=\frac{\partial F}{\partial x^{i}} .
$$

From the above considerations it follows that $F_{i j}=G^{i j}(y=\partial F / \partial x)$.

Our interest for complex coordinates on Sasakian toric manifolds comes from the fact that using this particular type of coordinates it is possible to express a complex volume form in a very convenient way. More exactly, on these manifolds the complex volume form $\Omega$ can be written as 17

$$
\Omega=e^{z^{1}} d z^{1} \wedge . . \wedge d z^{n} .
$$

Employing the above relation, in the next sections we show that is possible to extract the special Killing forms on manifolds of Sasaki-Einstein type.

\section{Complex volume form and foliated coordinates on geometric cone}

Within this section, we show that working with foliated coordinates we can locally extract the special Killing forms on a Sasaki-Einstein manifold.

More precisely, starting with the natural foliated structure on the metric cone $C(M)$ (which topologically can be identified with $\mathbb{R}_{>0} \times M$ ), we consider in the following the existence of (local) foliated coordinates $\left(r ; f^{2}, . ., f^{n}, \phi^{1}, . ., \phi^{n}\right) . r$ will stands for the transverse coordinate, while $f^{2}, . ., f^{n}, \phi^{1}, . ., \phi^{n}$ will stand for leafwise coordinates, describing the immersed submanifolds; for general topics concerning foliated structures we indicate 24. As the coordinates $\left(x^{i}, \phi^{i}\right)$ are assumed independent, the smooth link between the initial local coordinates $\left(x^{i}, \phi^{i}\right)$ and the foliated coordinates $\left(r ; f^{i}, \phi^{i}\right)$ is expressed in the form

$$
\left\{\begin{array}{l}
x^{i}=x^{i}\left(r, f^{2}, . ., f^{n}\right), \\
\phi^{i}=\phi^{i} .
\end{array}\right.
$$

Consequently, for the coframes $\left(d x^{i}, d \phi^{i}\right)$ and $\left(d r ; d f^{i}, d \phi^{i}\right)$ we get

$$
\left\{\begin{aligned}
d x^{i} & =\frac{\partial x^{i}}{\partial r} d r+\frac{\partial x^{i}}{\partial f^{j}} d f^{j} \\
d \phi^{i} & =d \phi^{i}
\end{aligned}\right.
$$


We consider also the Jacobi matrix

$$
\mathcal{A}:=\left(\begin{array}{llll}
\frac{\partial x^{1}}{\partial r} & \frac{\partial x^{1}}{\partial f^{2}} & \cdots & \frac{\partial x^{1}}{\partial f^{n}} \\
\vdots & \vdots & \vdots & \vdots \\
\frac{\partial x^{n}}{\partial r} & \frac{\partial x^{n}}{\partial f^{2}} & \cdots & \frac{\partial x^{n}}{\partial f^{n}}
\end{array}\right)
$$

In accordance with (3) (see also [20, 26]), we search for a complex differential form $\omega^{M}$ which verifies the relation

$$
\Omega=r^{n-1} d r \wedge \omega^{M}+\frac{r^{n}}{n} d \omega^{M} .
$$

The starting point is the following description of the complex volume form $\Omega$ using foliated coordinates

$$
\begin{aligned}
\Omega= & e^{z^{1}}\left(\frac{\partial x^{1}}{\partial r} d r+\frac{\partial x^{1}}{\partial f^{j}} d f^{j}+\mathrm{i} d \phi^{1}\right) \wedge \ldots \\
& \cdots \wedge\left(\frac{\partial x^{n}}{\partial r} d r+\frac{\partial x^{n}}{\partial f^{j}} d f^{j}+\mathrm{i} d \phi^{n}\right) .
\end{aligned}
$$

In order to extract $\omega^{M}$, we need to keep the trace of the differential form $d r$. We get $n$ terms in the above wedge product; considering $\Omega$ as a sum of differential forms of dimension $n$, for an arbitrary term let us assume that $i$ is the rank of $d r$, and in the left we have the ranks $j_{1}, . ., j_{p}$ where terms of type i $d \phi^{s}$ appear in the wedge product, while in the right of $d r$ we get also the ranks $k_{1}, . ., k_{q}$ where terms of type id $\phi^{s}$ also appear. Tracing out all the terms that appear, we finally get

$$
\omega^{M}=\frac{1}{r^{n-1}} e^{z^{1}} \sum_{i=1}^{n} \frac{\partial x^{i}}{\partial r} \sum_{\substack{p, q \\ C_{i}}} \mathrm{i}^{p+q} \sum_{\substack{J, K, L \\ C_{i, p, q}}}(-1)^{\mathcal{S}} \mathcal{A}_{J ; i ; K}^{L} d f^{L} \wedge d \phi^{J} \wedge d \phi^{K},
$$

where in the above expression we use multi-indices $J:=\left(j_{1}, . ., j_{p}\right)$ with $j_{1}<$ $. .<j_{p}, K:=\left(k_{1}, . ., k_{q}\right)$ with $k_{1}<. .<k_{q}$ and $L:=\left(l_{1}, . ., l_{n-p-q+1}\right)$ with $l_{1}<. .<l_{n-p-q+1}$; the condition $C_{i}$ means $1 \leq p \leq i-1$, and $i \leq q \leq n$, the condition $C_{i, p, q}$ means $1 \leq j_{1}<. .<j_{p}<i, i<k_{1}<. .<k_{q} \leq n$ and $2 \leq l_{1}<. .<l_{n-p-q+1} \leq n$. We also denote

$$
\begin{aligned}
d f^{L} & :=d f^{l_{1}} \wedge \cdots \wedge d f^{l_{n-p-q+1}} \\
d \phi^{J} & :=d \phi^{j_{1}} \wedge \cdots \wedge d \phi^{j_{p}} \\
d \phi^{K} & :=d \phi^{k_{1}} \wedge \cdots \wedge d \phi^{k_{q}}
\end{aligned}
$$

Furthermore, $\mathcal{A}_{J ; i ; K}^{L}$ stands for the determinant of the matrix obtained from $\mathcal{A}$ by suppressing the rows $j_{1}, \ldots, j_{p}, i, k_{1}, . ., k_{q}$, and selecting the columns $l_{1}, . ., l_{n-p-q+1}$; if no indices of a certain type exist, then we agree to denote this by 0 . For instance, for the term $\mathcal{A}_{J ; i ; K}^{0}$, which corresponds to the case when the term is constructed only using wedge product of differential forms of type $d \phi^{j_{s}}$ and 
$d \phi^{k_{s}}$, we agree to put $\mathcal{A}_{J ; i ; K}^{0}:=1$. Finally, concerning the sign of each term in the above sum, we have

$$
\begin{aligned}
\mathcal{S}= & n q-\sum_{s=1}^{q} k_{s}+n p-\sum_{s=1}^{p} j_{s} \\
& -\frac{q(q-1)}{2}-\frac{p(p-1)}{2}-p(q+1) .
\end{aligned}
$$

Remark 1. Let us notice that according to [20, Lemma 4.5], the differential form $\omega^{M}$ obtained from (4) will no longer depend on the transverse coordinate $r$. Consequently, $\omega^{M}$ will be a complex special Killing form on the manifold $M$, locally expressed in the coordinates $f^{2}, . ., f^{n}, \phi^{1}, . ., \phi^{n}$, while $\Xi:=\operatorname{Re} \omega^{M}$ and $\Upsilon:=\operatorname{Im} \omega^{M}$ will be real special Killing forms in the classical sense.

\section{An application: the spaces $Y^{p, q}$}

In the particular framework represented by the spaces $Y^{p, q}$, the special Killing forms were extracted by a direct calculation [26]. In this final section we present an alternative approach using the above results in this particular case.

Let us consider the explicit local metric of the 5-dimensional $Y^{p, q}$ manifold given by the line element [15]

$$
\begin{aligned}
d s^{2}= & \frac{1-c y}{6}\left(d \theta^{2}+\sin ^{2} \theta d \phi^{2}\right)+\frac{1}{w(y) q(y)} d y^{2}+\frac{q(y)}{9}(d \psi-\cos \theta d \phi)^{2} \\
& +w(y)\left[d \alpha+\frac{a c-2 y+c y^{2}}{6\left(a-y^{2}\right)}[d \psi-\cos \theta d \phi]\right]^{2},
\end{aligned}
$$

where

$$
\begin{aligned}
& w(y)=\frac{2\left(a-y^{2}\right)}{1-c y}, \\
& q(y)=\frac{a-3 y^{2}+2 c y^{3}}{a-y^{2}} .
\end{aligned}
$$

This metric is Einstein with $R i c_{g}=4 g$ for all values of the constants $a, c$. Moreover, the space is also Sasaki. For $c=0$, the metric takes the local form of the standard homogeneous metric on $T^{1,1}$ [15. Otherwise, the constant $c$ can be rescaled by a diffeomorphism, so in what follows we take $c=1$. For

$$
0<\alpha<1
$$

we can take the range of the angular coordinates $(\theta, \phi, \psi)$ to be $0 \leq \theta \leq 2 \pi, 0 \leq$ $\phi \leq 2 \pi, 0 \leq \psi \leq 2 \pi$. Choosing $0<a<1$, the roots $y_{i}$ of the cubic equation

$$
a-3 y^{2}+2 y^{3}=0
$$


are real, one negative $\left(y_{1}\right)$ and two positive $\left(y_{2}, y_{3}\right)$. If the smallest of the positive roots is $y_{2}$, one can take the range of the coordinate $y$ to be

$$
y_{1} \leq y \leq y_{2} .
$$

Following [15, 17], for this particular space we take the complex coordinates

$$
\begin{aligned}
z^{1}:= & \log \left(r^{3} \sin \theta \sqrt{\frac{p(y)(1-y)}{2}} e^{\mathrm{i} \psi}\right) \\
= & 3 \ln r+\ln \sin \theta+\frac{1}{2} \ln \frac{p(y)(1-y)}{2}+\mathrm{i} \psi^{\prime}, \\
z^{2}:= & \frac{1}{3 \sqrt{3}} \log \left(\tan \frac{\theta}{2} e^{\mathrm{i} \phi}\right) \\
= & \frac{1}{3 \sqrt{3}} \ln \tan \frac{\theta}{2}+\mathrm{i} \phi^{\prime}, \\
z^{3}:= & \frac{1}{6} \log \left(\frac{1}{\sin \theta} \sqrt{\left(y-y_{1}\right)^{-\frac{1}{y^{1}}}\left(y_{2}-y\right)^{-\frac{1}{y^{2}}}\left(y_{3}-y\right)^{-\frac{1}{y^{3}}}} e^{-6 \mathrm{i} \alpha-\mathrm{i} \psi}\right) \\
= & -\frac{1}{6} \ln \sin \theta-\frac{1}{2} \ln \left(\left(y-y_{1}\right)^{-\frac{1}{y^{1}}}\left(y_{2}-y\right)^{-\frac{1}{y^{2}}}\left(y_{3}-y\right)^{-\frac{1}{y^{3}}}\right) \\
& +\mathrm{i} \beta^{\prime},
\end{aligned}
$$

where we denote (see also [26])

$$
\begin{aligned}
p(y) & :=w(y) \cdot q(y)=\frac{2\left(a-3 y^{2}+2 y^{3}\right)}{a-y^{2}}, \\
\beta^{\prime} & :=-\alpha-\frac{1}{6} \psi, \\
\phi^{\prime} & :=\frac{1}{3 \sqrt{3}} \phi \\
\psi^{\prime} & :=\psi .
\end{aligned}
$$

Consequently

$$
\begin{aligned}
d \beta^{\prime} & =-d \alpha-\frac{1}{6} d \psi, \\
d \phi^{\prime} & =\frac{1}{3 \sqrt{3}} d \phi, \\
d \psi^{\prime} & =d \psi .
\end{aligned}
$$

From (8), we obtain

$$
\begin{aligned}
x^{1} & =3 \ln r+\ln \sin \theta+\frac{1}{2} \ln \frac{p(y)(1-y)}{2}, \\
x^{2} & =\frac{1}{3 \sqrt{3}} \ln \tan \frac{\theta}{2} \\
x^{3} & =-\frac{1}{6} \ln \sin \theta-\frac{1}{2} \ln \left(\left(y-y_{1}\right)^{-\frac{1}{y^{1}}}\left(y_{2}-y\right)^{-\frac{1}{y^{2}}}\left(y_{3}-y\right)^{-\frac{1}{y^{3}}}\right),
\end{aligned}
$$


while for the toric coordinates we simply have

$$
\begin{aligned}
\phi^{1} & =\psi^{\prime}, \\
\phi^{2} & =\phi^{\prime}, \\
\phi^{3} & =\beta^{\prime} .
\end{aligned}
$$

We derive from (8) the following useful relations (see also [15]).

$$
\begin{aligned}
d z^{1} & =\frac{\cos \theta}{\sin \theta} d \theta+3 \frac{d r}{r}-6 \frac{y}{p(y)} d y+\mathrm{i} d \psi^{\prime}, \\
d z^{2} & =\frac{1}{3 \sqrt{3}} \frac{1}{\sin \theta} d \theta+\mathrm{i} d \phi^{\prime}, \\
d z^{3} & =-\frac{1}{6} \frac{\cos \theta}{\sin \theta} d \theta+\frac{1}{p(y)} d y+\mathrm{i} d \beta^{\prime} .
\end{aligned}
$$

Now, comparing for instance with [15, (2.28)], it is easy to verify that

$$
\Omega=e^{z^{1}} d z^{1} \wedge d z^{2} \wedge d z^{3} .
$$

The above results, obtained in the general case of the metric cone of a SasakiEinstein manifold, can be now applied to calculate the special Killing forms in our particular case.

From the relation (11) and (12) it follows that we can consider the foliated coordinates $\left(r ; \theta, y, \psi^{\prime}, \phi^{\prime}, \beta^{\prime}\right)$; with respect to these convenient local coordinates, the Jacobi matrix $\mathcal{A}$ can be calculated as follows

$$
\mathcal{A}=\left(\begin{array}{ccc}
\frac{3}{r} & \frac{\cos \theta}{\sin \theta} & -\frac{6 y}{p(y)} \\
0 & \frac{1}{3 \sqrt{3}} \frac{1}{\sin \theta} & 0 \\
0 & -\frac{1}{6} \frac{\cos \theta}{\sin \theta} & \frac{1}{p(y)}
\end{array}\right)
$$

With the above consideration, we can now proceed to calculate the complex differential form $\omega^{M}$. First of all we have $\frac{\partial x^{1}}{\partial r} \neq 0$, all other terms of this type vanish, so the only possibility for $i$ is 1 ; consequently $p=0$, there is no $j_{s}$, and $q$ can only be 0,1 or 2 .

Using the relation (5), we get

$$
\begin{aligned}
\omega^{M}= & \frac{1}{r^{2}} \frac{3}{r}\left(r^{3} \sqrt{\frac{p(y)(1-y)}{2}} \sin \theta\right) e^{\mathrm{i} \psi^{\prime}} \\
& \times\left((-1)^{0} \mathrm{i}^{0} \mathcal{A}_{0 ; 1 ; 2}^{23} d \theta \wedge d y+(-1)^{1} \mathrm{i}^{1} \mathcal{A}_{0 ; 1 ; 2}^{2} d \theta \wedge d \phi^{\prime}\right. \\
& +(-1)^{1} \mathrm{i}^{1} \mathcal{A}_{0 ; 1 ; 2}^{3} d y \wedge d \phi^{\prime}+(-1)^{0} \mathrm{i}^{1} \mathcal{A}_{0 ; 1 ; 3}^{2} d \theta \wedge d \beta^{\prime} \\
& \left.(-1)^{0} \mathrm{i}^{1} \mathcal{A}_{0 ; 1 ; 3}^{3} d y \wedge d \beta^{\prime}+(-1)^{0} \mathrm{i}^{2} \mathcal{A}_{0 ; 1 ; 2,3}^{0} d \phi^{\prime} \wedge d \beta^{\prime}\right) .
\end{aligned}
$$

We calculate the coefficients in the following. 
For $q=0$ we obtain

$$
\begin{aligned}
& \mathcal{A}_{0 ; 1 ; 2}^{23}=\left|\begin{array}{cc}
\frac{1}{3 \sqrt{3}} \frac{1}{\sin \theta} & 0 \\
-\frac{1}{6} \frac{\cos \theta}{\sin \theta} & \frac{1}{p(y)}
\end{array}\right| \\
& =\frac{1}{3 \sqrt{3}} \frac{1}{\sin \theta p(y)} \text {. }
\end{aligned}
$$

Next, for $q=1$ one can calculate

$$
\begin{array}{ll}
\mathcal{A}_{0 ; 1 ; 2}^{2}=-\frac{1}{6} \frac{\cos \theta}{\sin \theta}, & \mathcal{A}_{0 ; 1 ; 2}^{3}=\frac{1}{p(y)}, \\
\mathcal{A}_{0 ; 1 ; 3}^{2}=\frac{1}{3 \sqrt{3}} \frac{1}{\sin \theta}, & \mathcal{A}_{0 ; 1 ; 3}^{3}=0 .
\end{array}
$$

Finally, for $q=2$ we obtain

$$
\mathcal{A}_{0 ; 1 ; 2,3}^{0}=1 .
$$

Plugging now all the above terms in the formula (13), we end up with

$$
\begin{aligned}
\omega^{M}= & e^{\mathrm{i} \psi^{\prime}} \sqrt{\frac{1-y}{6 p(y)}}\left(d \theta \wedge d y+\mathrm{i} \frac{\sqrt{3}}{2} \cos \theta p(y) d \theta \wedge d \phi^{\prime}\right. \\
& -\mathrm{i} 3 \sqrt{3} \sin \theta d y \wedge d \phi^{\prime}+\mathrm{i} p(y) d \theta \wedge d \beta^{\prime} \\
& \left.-3 \sqrt{3} p(y) \sin \theta d \phi^{\prime} \wedge d \beta^{\prime}\right)
\end{aligned}
$$

We write $\omega^{M}$ in a more convenient form,

$$
\begin{aligned}
& \omega^{M}= \sqrt{\frac{1-y}{6 p(y)}}\left(\cos \psi^{\prime}+\mathrm{i} \sin \psi^{\prime}\right) \times\left(\left[-d y \wedge d \theta+3 \sqrt{3} p(y) \sin \theta d \beta^{\prime} \wedge d \phi^{\prime}\right]\right. \\
&\left.+\mathrm{i}\left[-p(y) d \beta^{\prime} \wedge d \theta+\frac{\sqrt{3}}{2} \cos \theta p(y) d \theta \wedge d \phi^{\prime}-3 \sqrt{3} \sin \theta d y \wedge d \phi^{\prime}\right]\right) .
\end{aligned}
$$

From here, calculating the real and imaginary part, we get

$$
\begin{aligned}
\Xi= & \sqrt{\frac{1-y}{6 p(y)}}\left(\cos \psi^{\prime}\left[-d y \wedge d \theta+3 \sqrt{3} p(y) \sin \theta d \beta^{\prime} \wedge d \phi^{\prime}\right]\right. \\
& -\sin \psi^{\prime}\left[-3 \sqrt{3} \sin \theta d y \wedge d \phi^{\prime}-p(y) d \beta^{\prime} \wedge d \theta\right. \\
& \left.\left.+\frac{\sqrt{3}}{2} \cos \theta p(y) d \theta \wedge d \phi^{\prime}\right]\right),
\end{aligned}
$$


and respectively

$$
\begin{aligned}
\Upsilon= & \sqrt{\frac{1-y}{6 p(y)}}\left(\operatorname { c o s } \psi ^ { \prime } \left[-3 \sqrt{3} \sin \theta d y \wedge d \phi^{\prime}-p(y) d \beta^{\prime} \wedge d \theta\right.\right. \\
& \left.+\frac{\sqrt{3}}{2} \cos \theta p(y) d \theta \wedge d \phi^{\prime}\right]+\sin \psi^{\prime}[-d y \wedge d \theta \\
& \left.\left.+3 \sqrt{3} p(y) \sin \theta d \beta^{\prime} \wedge d \phi^{\prime}\right]\right) .
\end{aligned}
$$

Replacing now $\psi^{\prime}, \phi^{\prime}, \beta^{\prime}$ by $\psi, \phi, \beta$ and considering also (9) and (10), we eventually obtain (14) and (15) from [26].

\section{Conclusions}

It is well-known that a lot of examples of Sasaki-Einstein manifolds may be obtained via toric geometry, and such examples are a good testing ground for the predictions of the AdS/CFT correspondence [17. In this paper we give a general scheme to construct the special Killing forms on a toric Sasaki-Einstein manifold. This procedure is effectively exemplified in the case of the $Y^{p, q}$ manifolds, and computations in this explicit 5-dimensional space reveal that the special Killing forms obtained in this article agree with the results previously obtained in [26] with a different approach.

On the other hand, in [16] the authors investigated two explicit infinite families $Y^{p, q}$ of Sasaki-Einstein 7-manifolds, which are lens-space bundles $S^{3} / \mathbb{Z}_{p}$ over $C P^{2}$ and $C P^{1} \times C P^{1}$, respectively, and showed that the metric cones over these Sasaki-Einstein 7-manifolds are in fact toric. It is the hope of the authors that the techniques developed in this paper can be applied to extract the special Killing forms on the 7-dimensional $Y^{p, q}$ spaces, as well on the $L^{a b c}$ spaces. We note that the spaces $L^{a b c}$ (where $a, b, c$ are three positive coprime integers that satisfy some other relations), are certain 5-dimensional EinsteinSasaki manifolds with a $T^{3}$-worth of isometries acting with cohomogeneity two [4. These metrics are constructed starting by rotating anti-de Sitter black hole metrics in 5 dimensions, Euclideanizing, and then taking an appropriate limit motivated by physical considerations. The restrictions on $a, b, c$ arise from requiring that the locally defined metrics extend smoothly over a compact, nonsingular 5-manifold. When $a+b=2 c$, these metrics reduce to the cohomogeneity one metrics $Y^{p, q}$.

\section{Acknowledgments}

The authors would like to thank the referee for his valuable comments and suggestions which helped to improve the paper. M. Visinescu was supported by CNCS-UEFISCDI, project number PN-II-ID-PCE-2011-3-0137. The work 
of G.E. Vîlcu was supported by CNCS-UEFISCDI, project number PN-II-IDPCE-2011-3-0118.

\section{References}

[1] M. Abreu, Kähler geometry of toric manifolds in symplectic coordinates, to appear in Toric Varieties in Algebraic Geometry and Physics, V. Batyrev (ed.), AMS, arXiv:math.DG/0004122.

[2] C. Boyer and K. Galicki, 3-Sasakian manifolds, Surveys Diff. Geom. 7 (1999) 123-184; arXiv:hep-th/9810250.

[3] C. Boyer and K. Galicki, On Sasakian-Einstein geometry, Internat. J. Math. 11 (2000) 873-909; arXiv:math/9811098.

[4] W. Chen, M. Cvetic, H. Lü, C. N. Pope, J. F. Vázquez-Poritz, Resolved Calabi-Yau cones and flows from $L^{\text {abc }}$ superconformal field theories, Nuclear Phys. B 785 (2007), 74-97; arXiv:hep-th/0701082.

[5] K. Cho, A. Futaki and H. Ono, Uniqueness and examples of compact toric Sasaki-Einstein metrics, Commun. Math. Phys. 277 (2008) 439458; arXiv:math/0701122.

[6] V. P. Frolov and D. Kubiznak, Higher-dimensional black holes: hidden symmetries and separation of variables, Class. Quantum Grav. 25 (2008) 154005; arXiv: 0802.0322.

[7] A. Futaki, H. Ono and G. Wang, Transverse Kähler geometry of Sasaki manifolds and toric Sasaki-Einstein manifolds, J. Diff. Geometry. 83 (2009) 585-636; arXiv:math/0607586.

[8] J. P. Gauntlett, D. Martelli, J. Sparks and D. Waldram, SasakiEinstein metrics on $S^{2} \times S^{3}$, Adv. Theor. Math. Phys. 8 (2004) 711734; arXiv:hep-th/0403002.

[9] J. P. Gauntlett, D. Martelli, J. Sparks and D. Waldram, A New Infinite Class of Sasaki-Einstein Manifolds, Adv. Theor. Math. Phys. 8 (2004) 987-1000; arXiv:hep-th/0403038.

[10] V. Guillemin, Kaehler structures on toric varieties, J. Diff. Geom. 40 (1994) 285-309.

[11] V. Guillemin, Moment maps and combinatorial invariants of Hamiltonian $T^{n}$-spaces, Progress in Mathematics, Volume 122, Birkhäuser, Boston (1994).

[12] T. Houri, H. Takeuchi, Y. Yasui, A deformation of Sasakian structure in the presence of torsion and supergravity solutions, Class. Quant. Grav. 30 (2013), no. 13, 135008, 31 pp; arXiv:hep-th/1207.0247. 
[13] T. Kashiwada, A note on a Riemannian space with Sasakian 3structure, Natur. Sci. Rep. Ochanomizu Univ. 22 (1971) 1-2.

[14] J. M. Maldacena, The large $N$ limit of superconformal field theories and supergravity, Adv. Theor. Math. Phys. 2 (1998) 231-252.

[15] D. Martelli and J. Sparks, Toric geometry, Sasaki-Einstein manifolds and a new infinite class of Ads/CFT duals, Commun. Math. Phys. 262 (2006) 51-89; arXiv:hep-th/0411238.

[16] D. Martelli and J. Sparks, Notes on toric Sasaki-Einstein sevenmanifolds and AdS4/CFT3, J. High Energy Phys. 11 (2008) 016; arXiv:0808.0904.

[17] D. Martelli, J. Sparks and S.-T. Yau, The geometric dual of amaximisation for toric Sasaki-Einstein manifolds, Commun. Math. Phys. 268 (2006) 39-65; arXiv:hep-th/0503183.

[18] D. Martelli, J. Sparks and S.-T. Yau, Sasaki-Einstein manifolds and volume minimisation, Comm. Math. Phys. 280 (2008) 611-673; arXiv:hep-th/0603021.

[19] A. Moroianu, Lectures on Kähler Geometry, Cambridge University Press, 2007.

[20] U. Semmelmann, Conformal Killing forms on Riemannian manifolds, Math. Z. 245 (2003) 503-527; arXiv:math.DG/0206117.

[21] J. Sparks, Sasaki-Einstein manifolds, Surv. Diff. Geom. 16 (2011) 265324; $\operatorname{arXiv:1004.2461.}$

[22] C. Stromenger, Sasakian manifolds: Differential Forms, Curvature and Conformal Killing Forms, Ph.D. Thesis, University of Köln, 2011.

[23] S. Tachibana and W. N. Yu, On a Riemannian space admitting more than one Sasakian structures, Tôhoku. Math. J. 22 (1970) 536-540.

[24] Ph. Tondeur, Geometry of Foliations, Birkhäuser, Basel, Boston, 1997.

[25] C. van Coevering, A construction of complete Ricci-flat Kähler manifolds, arXiv:0803.01112.

[26] M. Visinescu, Killing forms on the five dimensional EinsteinSasaki $Y(p, q)$ spaces, Mod. Phys. Lett. A 27 (2012) 1250217; arXiv:1207.2581.

[27] M. Visinescu and G. E. Vîlcu, Hidden symmetries of Euclideanised Kerr-NUT-(A)dS metrics in certain scaling limits, SIGMA 8 (2012) 058; arXiv:1205.6036.

[28] H.-U. Yee, AdS/CFT with Tri-Sasakian Manifolds, Nuclear Phys. B 774 (2007), 232-255; arXiv:hep-th/0612002. 